\title{
In-situ Microstructural Observations and Micro-grid Analyses of Transfomation Superplasticity in Pure Iron*
}

\author{
By Yasunori SAOTOME** and Nobuhiro IGUCHI***
}

\section{Synopsis}

In the present paper, the microstructural changes associated with phase transformation and the straining behavior in polycrystalline structures dueing transformation superplastic deformation are investigated. In-situ observations of microstructural changes during A $c_{3}$ transformation on rapid heating in pure iron have been carried out with a high temperature optical microscope and a dark field reflection high temperature microscope. Both of hot-stage microscopes were specially designed for this study. The distribution of superplastic strain has been examined by microscopic strain analyses by using a micro-grid pattern with $12.7 \mu \mathrm{m}$ intervals. The main results are as follows:

(1) Ac $c_{3}$ transformation process at a heating rate under $50 \mathrm{~K} / \mathrm{s}$ is predominantly nucleation of austenite grains at the prior ferrite grain boundaries and triple points. A subsequent growth of grains into the prior ferrite matrices is observed. The growth of austenite grains is not always isotropic under a tensile stress.

(2) In the initial stage of transformation, superplastic strain is induced by the sliding at $\gamma / \alpha$ transformation interface along the prior ferrite grain boundaries. The observed superplastic strain is also associated with the grain rotation, corresponding to the growth of austenite grains which surround ferrite grains.

(3) In the intermediate stage of transformation, the sliding deformation is generated at the migrating transformation interface associated with the growth of austenite grains. Accumulated strain by the sliding is left within the previously transformed region.

(4) These observations suggest that the sliding mechanism at the migrating transformation interface is a principal mechanism of transformation superplasticity.

Key words: transformation superplasticity; iron; in-situ observation; grid analysis; high temperature microscope.

\section{Introduction}

In previous papers, macroscopic deformation due to transformation superplasticity was analyzed in pure iron ${ }^{11}$ and low carbon steels ${ }^{2)}$ under rapid heating and cooling cycles. With a micro-grid pattern spaced at $12.7 \mu \mathrm{m}$ intervals, semi-macroscopic analyses have been performed on the superplastically deformed pure iron. ${ }^{3)}$ It was found that (i) the deformation was principally incompressible and homogeneous and (ii) inhomogeneous distribution of strain along with a rotation component was observed.

\section{Experimental Procedures}

\section{High Temperature Microscope}

A high temperature microscope was developed specially for the observation of superplastic deformation and microstructural changes during transformation processes. A schematic diagram of the newly devel- oped apparatus is shown in Fig. 1; the main functions are application of rapid heating and cooling cycles and loading of static tension to the specimen. The additional facilities are a chamber for atmospheric control, a supply of etchant for specimen surface, an optical microscope, a motor driven camera, and a pen-recorder.

The chemical analysis and the specimen dimension are shown in Table 1 and Fig. 2, respectively. The specimens were heated to a predetermined tempera-

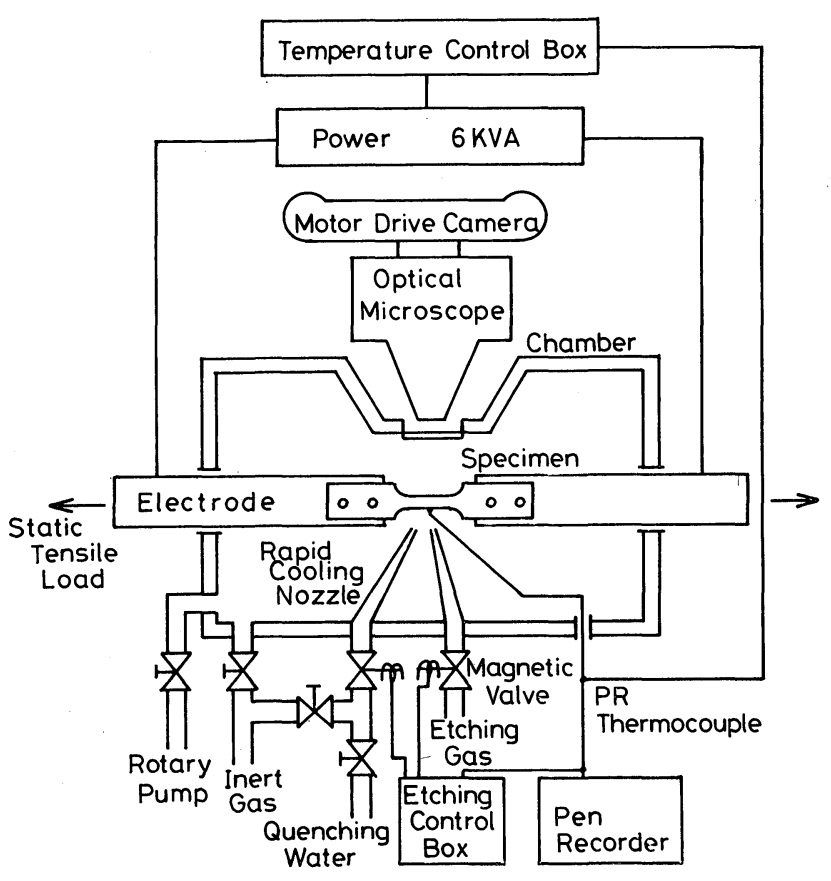

Fig. 1. Schematic diagram of test apparatus.

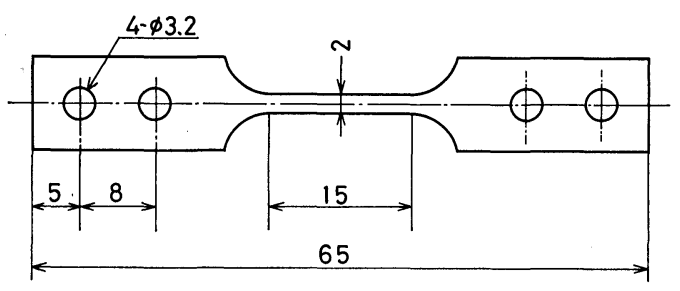

Fig. 2. Specimen dimension.

Table 1. Chemical composition. (wt \%)

\begin{tabular}{cccccc}
\hline & $\mathrm{G}$ & $\mathrm{Si}$ & $\mathrm{Mn}$ & $\mathrm{P}$ & $\mathrm{S}$ \\
\hline Pure iron & 0.017 & 0.036 & 0.18 & 0.014 & 0.010 \\
\hline
\end{tabular}

\footnotetext{
* Manuscript received on February 5, 1987; accepted in the final form on May 15, 1987. (C) 1987 ISIJ

** Faculty of Engineering, Gumma University, Tenjin-cho, Kiryu 376.

*** School of Science and Engineering, Waseda University, Ohkubo, Shinjuku-ku, Tokyo 160.
} 
ture at a rate up to $200 \mathrm{~K} / \mathrm{s}$ with resistance heating by using a $6 \mathrm{kVA}$ transformer which was regulated. with a current phase controller, and were cooled in the chamber at a selected rate by controlled jet of argon gas from a cooling nozzle. The specimen temperature was regulated by means of feedback control from the thermo-electromotive force of PR thermocouple spot-welded to the center of a specimen. The force was simultaneously recorded on a pen-recorder. Hence, selection of thermal cycles between a temperature interval can be achieved and an isothermal soaking after rapid heating can be also performed.

The specimen was contained in an argon or vacuum atmosphere inside the chamber, and subjected to a constant tensile load through the electrodes. The apparatus is equipped with an instantaneous etching system operated by triggering a magnetic valve of etching gases to reveal clearly the outline of grain boundaries or interfaces undergoing transformation and a dark field reflection high temperature microscope that exposes dynamically the surface relief due to transformation (see Fig. 3).

With this novel system, the microstructural changes during transformation superplastic deformation and the straining behavior were observed directly and concurrently on the same microscopic field of view and were recorded on $16-\mathrm{mm}$ cine-films or $35-\mathrm{mm}$ films by using a motor driven camera.

\section{Strain Analysis with Micro-grid Technique}

A grid analysis was applied to clarify the characteristic deformation behavior associated with transformation superplasticity in a sub-macroscopic scale. The strain distribution in polycrystalline structures can be analyzed by measuring the displacement of each grid point which is inprinted on the surface of specimens by photo-etching technique. The spacing of the fiducial grid points corresponds to a gauge length in strain measurements. In the present study, the interval was selected to be $12.7 \mu \mathrm{m}$ (2 000 lines/ inch in density) in order to detect the local deforma-

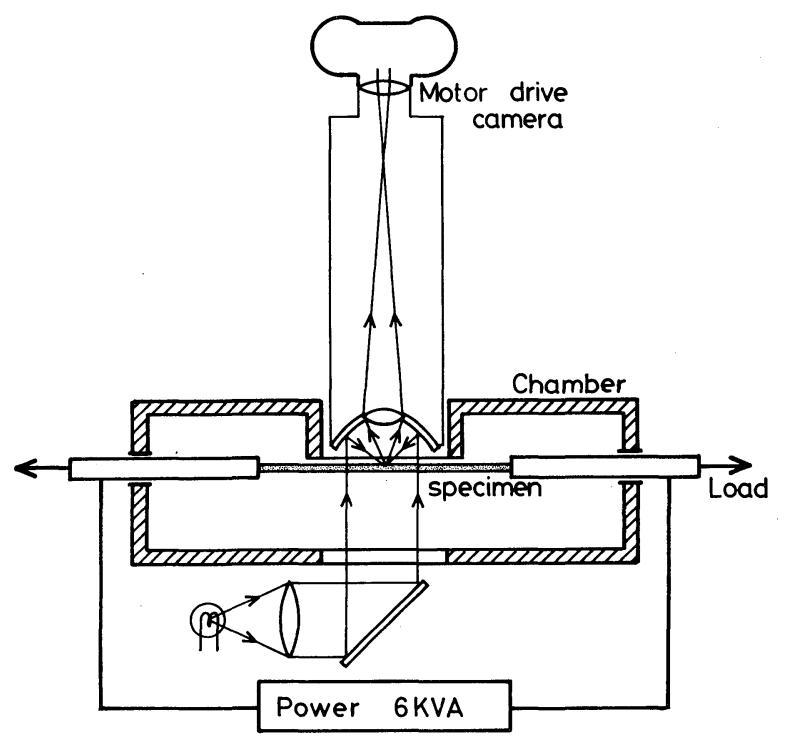

Fig. 3. Dark field reflection high temperature microscope. tion behavior at and near the transformation interface. The process of the photo-etching is shown in Fig. 4 .

The deformation behavior of specimen was observed on the same field of microscopic observation and simultaneously recorded on $35-\mathrm{mm}$ films. In the strain analysis, the displacement of each grid was measured by magnification of the images on films with a laboratory automation system (see Fig. 5). Strain distributions were calculated and displayed on an $X-Y$ plotter.

\section{Results}

1. In-situ Observation of Microstructural Changes during $A c_{3}$ Transformation during Rapid Heating

\section{Structural Changes under No Load}

In-situ observation of $A c_{3}$ transformation during rapid heating was carried out by the apparatus (the dark field reflection high temperature microscope with 35-mm motor driven camera). Figure 6 represents a sequence of films for four temperatures. The results indicate that the initiation of $A c_{3}$ transformation occurs by formation of austenite grains at the prior ferrite grain boundary or triple points at a heating rate of under $20 \mathrm{~K} / \mathrm{s}$ followed by complete austenitizing. Without loading, the austenite grains are found to grow isotropically. Figure 7 shows the geometrical relationship between the prior ferrite grain boundaries and the contour of resultant austenite grain bound-

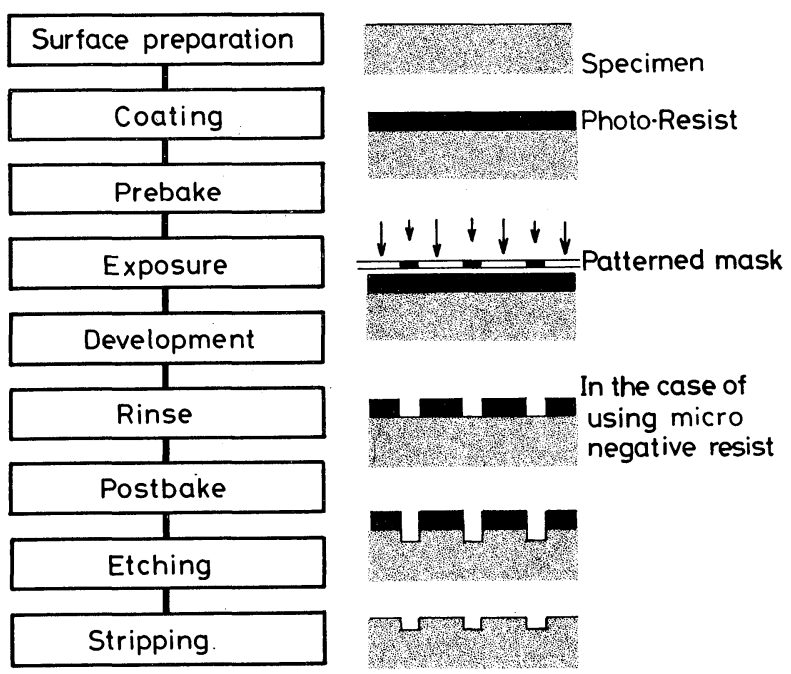

Fig. 4. Technique of photo-etching of grid pattern on specimen surface.

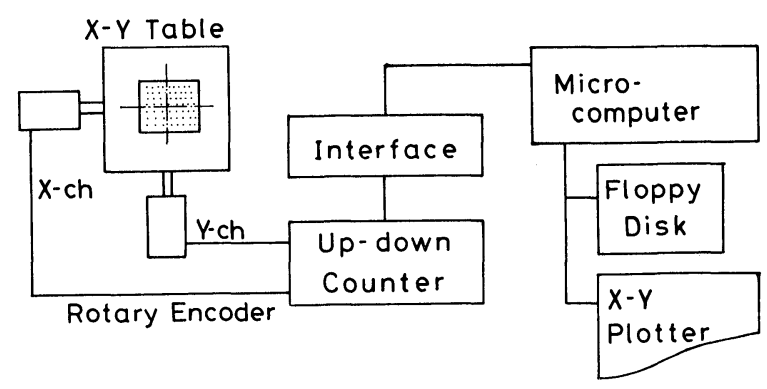

Fig. 5. Laboratory automation system for grid analysis. 


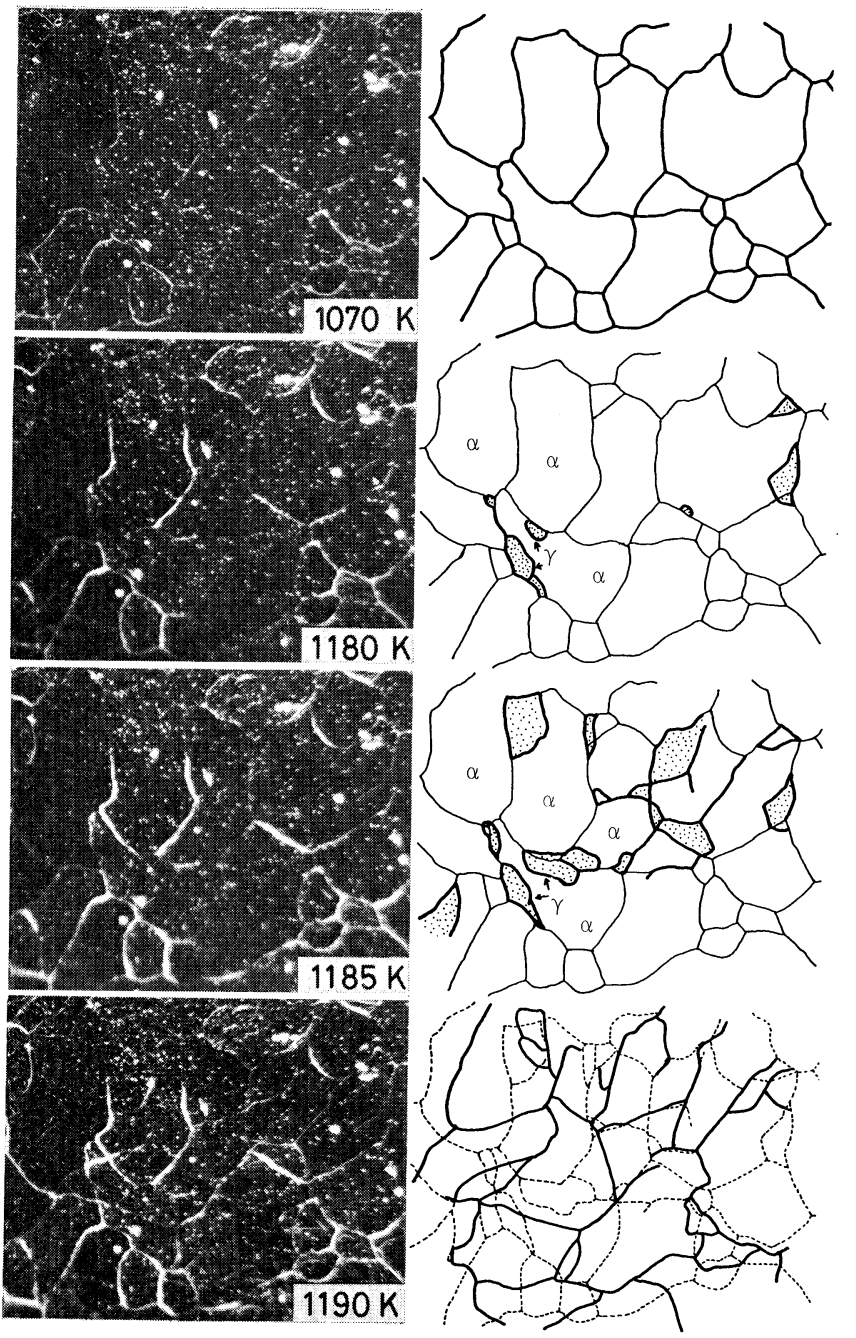

Fig. 6. In-situ observation of $A c_{3}$ transformation process at a heating rate of $20 \mathrm{~K} / \mathrm{s}$ in pure iron without loading by dark field reflection high temperature microscope. aries.

\section{Structural Changes under Tensile Loading}

Transformation superplastic deformation is generated under an applied load. Direct observation of the movement of transformation interfaces was carried out. The results are shown in Fig. 8.4) Although the transformation proceeds similarly as without loading, austenite grains are found to grow anisotropically into ferrite matrix under loading of a tensile stress of

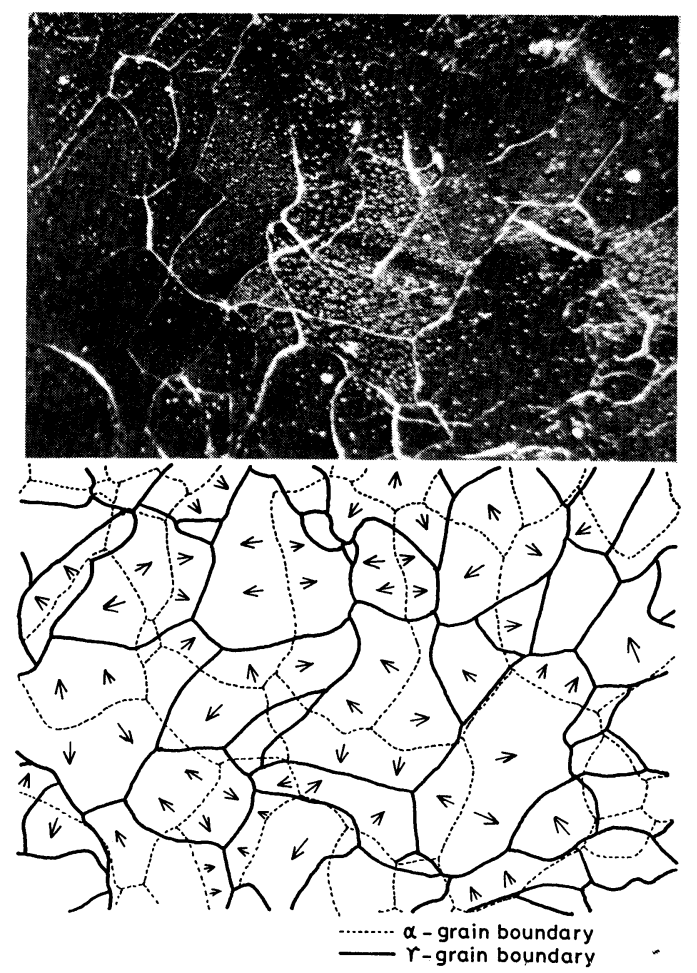

Fig. 7. Relation between austenite grain boundaries after $A c_{3}$ transformation and prior ferrite grain boundaries.

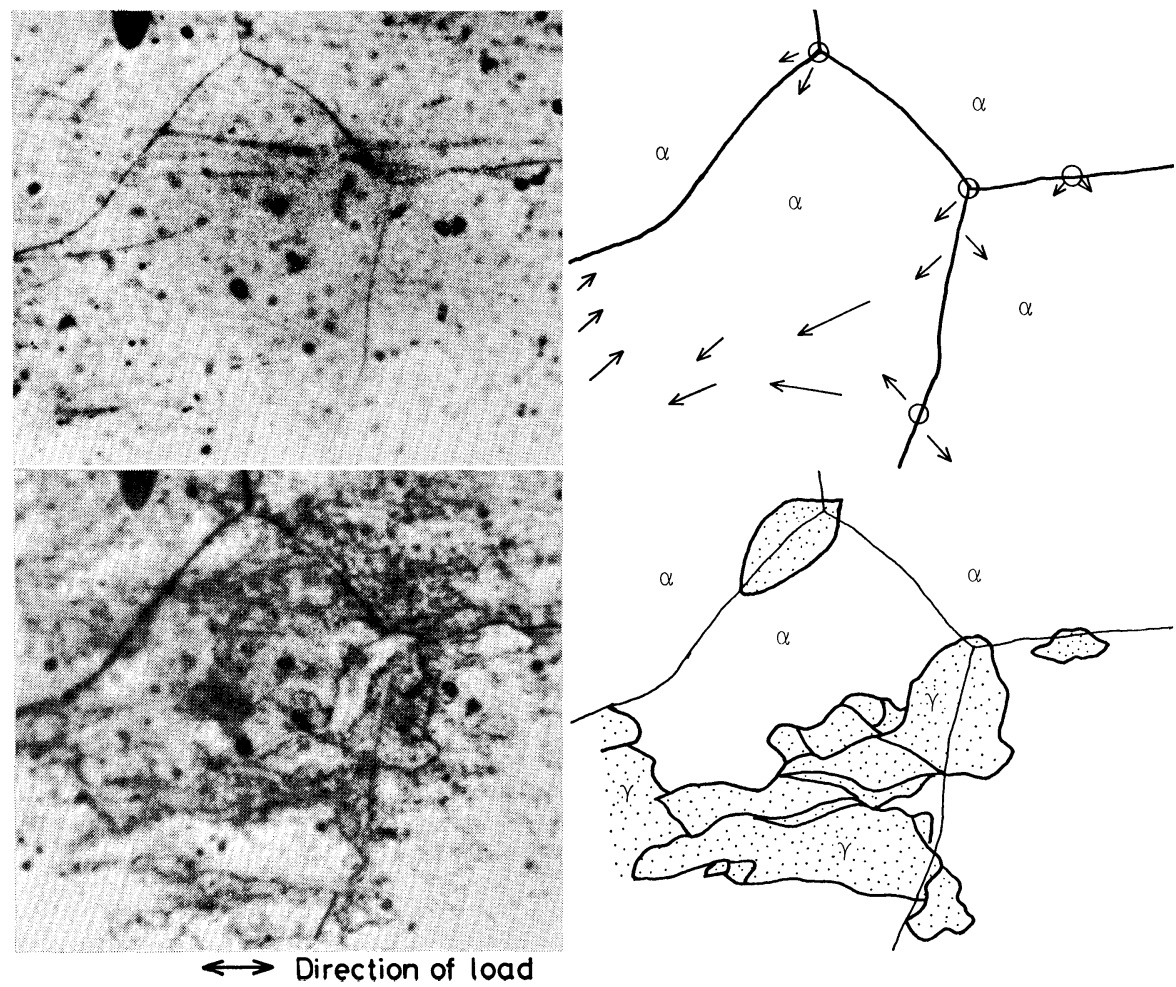

Fig. 8.

In-situ observation of $A c_{3}$ transformation process in pure iron at a heating rate of $5 \mathrm{~K} / \mathrm{s}$ under a tensile stress of $5 \mathrm{MPa}$. 
Fig. 9.

Morphological changes on specimen surface (a) before and (b) after heating to a temperature just above $A c_{3}$ s point under a tensile stress of $20 \mathrm{MPa}$, (c) same as (b), (d) $\alpha-\gamma-\alpha$ transformed region identified with (a) and (b).
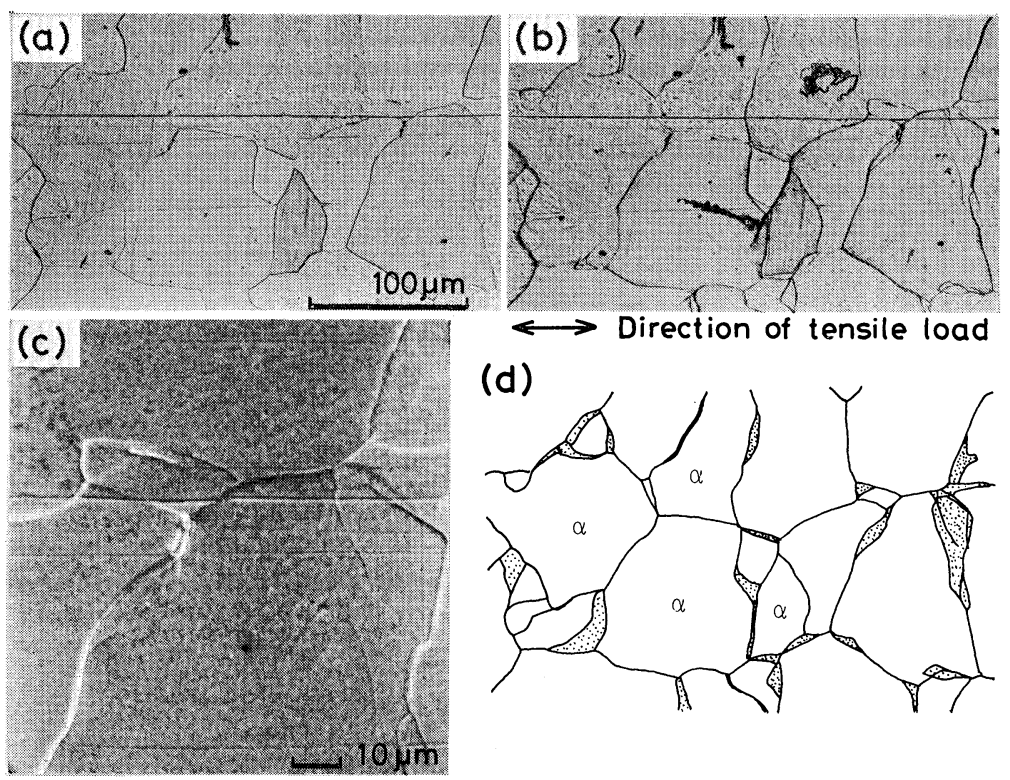

$5 \mathrm{MPa}$.

\section{Observations of Surface Morphology after Rapid Cooling from Transformation Temperature}

When a specimen is rapidly heated to a temperature just above the transformation start $\left(A c_{3} s\right)$ point and then rapidly cooled, both of $\alpha-\gamma$ and $\gamma-\alpha$ transformation proceed simultaneously in a limited region of polycrystalline structures. The structure before heating, as shown in Fig. 9(a), exhibits the ferrite grain boundaries after light etching. Figure 9(b) shows the microstructure observed at room temperature after the thermal history. In comparison with Fig. 9(a), variations are found locally in the proximity to ferrite grain boundaries. These variations were shown to be slight surface undulations formed during the cycle, as shown in a scanning micrograph of Fig. 9 (c). The morphological changes of surface will be associated with partial austenitizing. Figure 9(d) shows the feature of $\alpha-\gamma$ transformation fronts at an instant at the temperature $T_{\max }$. Hence, the process of austenite grain growth during rapid heating can be determined. To clarify the influence of applied stress on the undulation, further experiments were carried out. Without loading, a slight undulation is found on the specimen surface, as shown in Fig. 10(a). The undulation will correspond to dilatation which is generally useful to analyze the process of phase transformation. Under a tensile stress, transformation superplastic deformation is generated and simultaneously a remarkable undulation occurs. When the stress is above the critical stress of superplasticity, $\sigma_{c}=20$ $\mathrm{MPa},{ }^{3)}$ the induced deformation will be similar to creep deformation ${ }^{1,3)}$ and reliefs caused by slip is noticed within grain interior (Figs. $10(\mathrm{~d})$ and $10(\mathrm{e})$ ). On the contrary, if the stress is below the critical stress, slip lines in grain interior can not be found in Fig. 10 (c), although the offset at transformation front and slight rotations of each austenite grain are recognized. The fronts of austenite grains migrate anisotropically but smoothly into prior ferrite matrix, and the interfaces of austenite and ferrite interface appear straight.

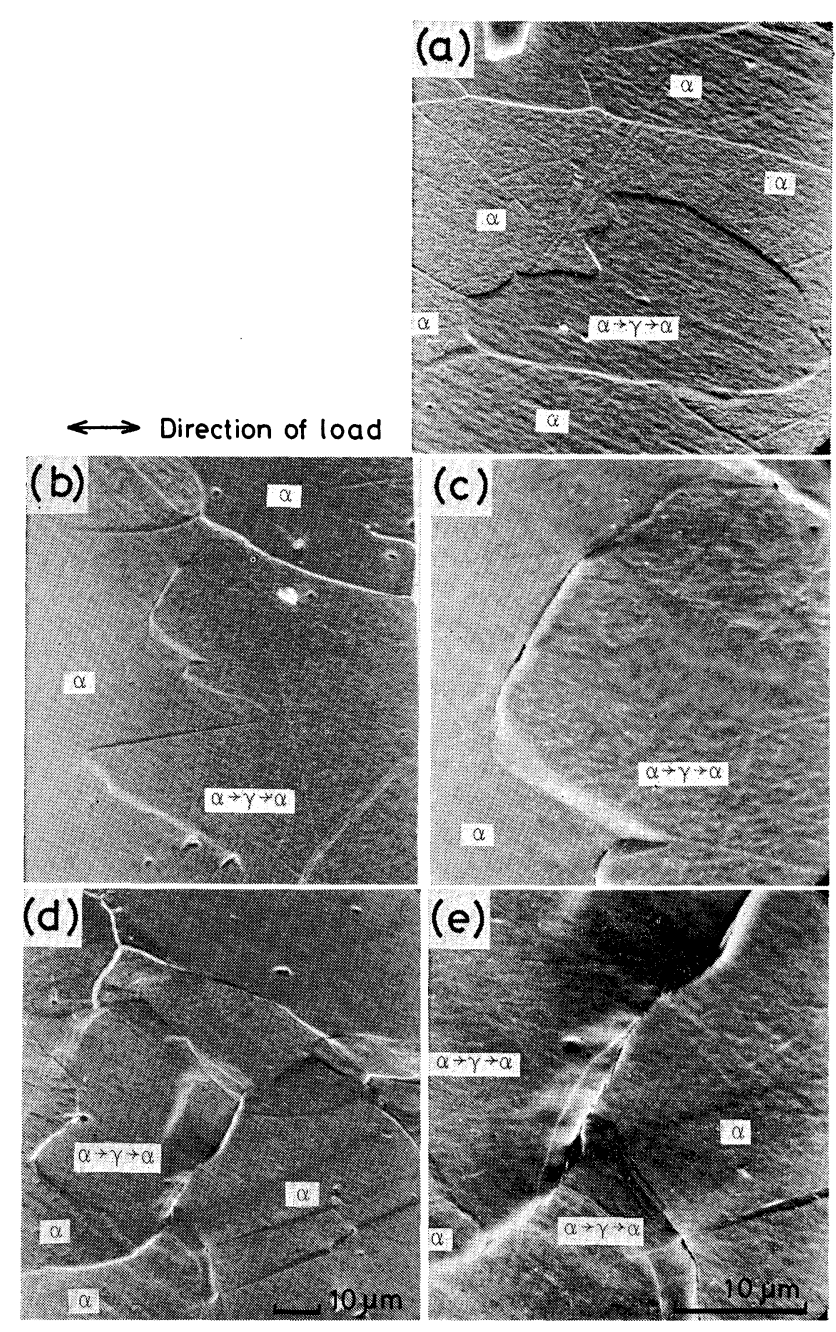

Fig. 10. Morphological changes on the specimen surface after heating to a temperature above $A c_{3}$ s point under various tensile stress (a) unloading, ((b), (c)) $20 \mathrm{MPa}$, and ((d), (e)) $30 \mathrm{MPa}$.

\section{Strain Analysis of Superplastic Deformation}

The relation between dilatation at $A c_{3}$ transformation under rapid heating and superplastic deforma- 
tion behavior under a torsional stress, was investigated by using a previously reported apparatus. ${ }^{1)}$ Both phenomena are associated with the microstructural changes, as shown in Fig. 11. The process will be classified into the following three stages.

(I) Initial stage: nucleation of austenite grains at prior ferrite grain boundary or triple point and the subsequent grain growth

(II) Intermediate stage: the growth of austenite grains

(III) Final stage: coalescence of austenite grains to form stable austenite grain boundaries

The strain analyses were carried out at each stage to study how the superplastic straining propagates in polycrystalline structures. The specimen surface was buff-polished and then slightly etched to reveal ferrite grain boundaries. An orthogonal fiducial grid pattern with a spacing of $12.7 \mu \mathrm{m}$ was photo-etched on the specimen surfaces. When the specimen was heated rapidly to $T_{\max }$ in the $A c_{3}$ transformation temperature range, the transformation process $(\mathrm{I}),(\mathrm{I}+$ II), or $(\mathrm{I}+\mathrm{II}+\mathrm{III})$ proceeded by accompanying superplastic deformation. On cooling from $T_{\max }$, superplastic deformation was induced along with the progress of $\alpha-\gamma$ transformation. After completion of the temperature cycle, the specimen exhibits the traces of transformation fronts produced by the strain generated under partial $\alpha-\gamma$ and $\gamma-\alpha$ transformation at $T_{\max }$.

1. Straining Behavior at the Initial Stage of Transformation

A specimen was rapidly heated at a rate of $50 \mathrm{~K} / \mathrm{s}$ up to the temperature just above $A c_{3}$ s under a tensile

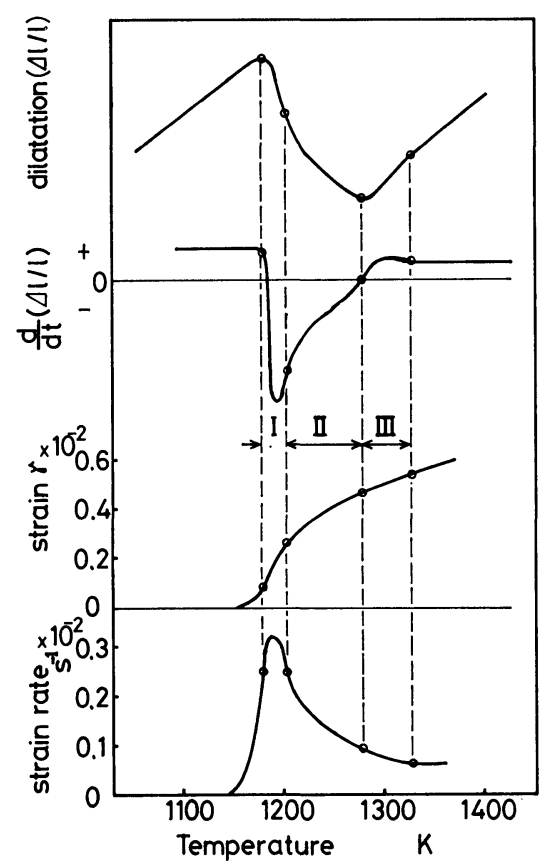

Fig. 11. Relation between the dilatometric change and superplastic deformation during $A c_{3}$ transformation in pure iron: heating rate $=50 \mathrm{~K} / \mathrm{s}$; shear stress = $4 \mathrm{MPa}$. stress, and then rapidly cooled. The thermal and strain histories are shown in Fig. 12(a). Figure 13(a) shows the appearance of the specimen surface; the traces of $\alpha-\gamma-\alpha$ transformation in the vicinity of prior ferrite grain boundaries are represented in dotted zones. With reference to straining behavior, displacements are noticed at the fiducial grid points which are located on the prior ferrite grain boundaries. This indicates that grain boundary slide is a predominant process along the prior ferrite grain boundaries. Reference lines are drawn by interpolation the grid points as shown in Fig. 13(b), to make clear the sliding behavior. The rotation of each ferrite grain is detected by measuring the angle between a grid line of grain interior and the tensile direction. The rotation is found to be a result of the accumulation of slides along the periphery of ferrite grain. These characteristics in the vicinity of grain boundaries were investigated by a scanning electron microscope. In the microphotographs of Fig. 14, the traces of localized transformation along with slip deformation are recognized in the vicinity of prior ferrite grain boundaries. Especially in Fig. 14(c), the undulated zone along the prior ferrite grain boundary suggests that an extensive slip deformation has taken place in association with the progress of $\alpha-\gamma-\alpha$ transformation in the area.

\section{Straining Behavior at the Intermediate Stage of Trans-} formation

Strain analysis was carried out to see the migration behavior of transformation interfaces accompanied with growth of austenite grains. A specimen was rapidly heated at about $20 \mathrm{~K}$ above $A c_{3}$ s point (Fig.

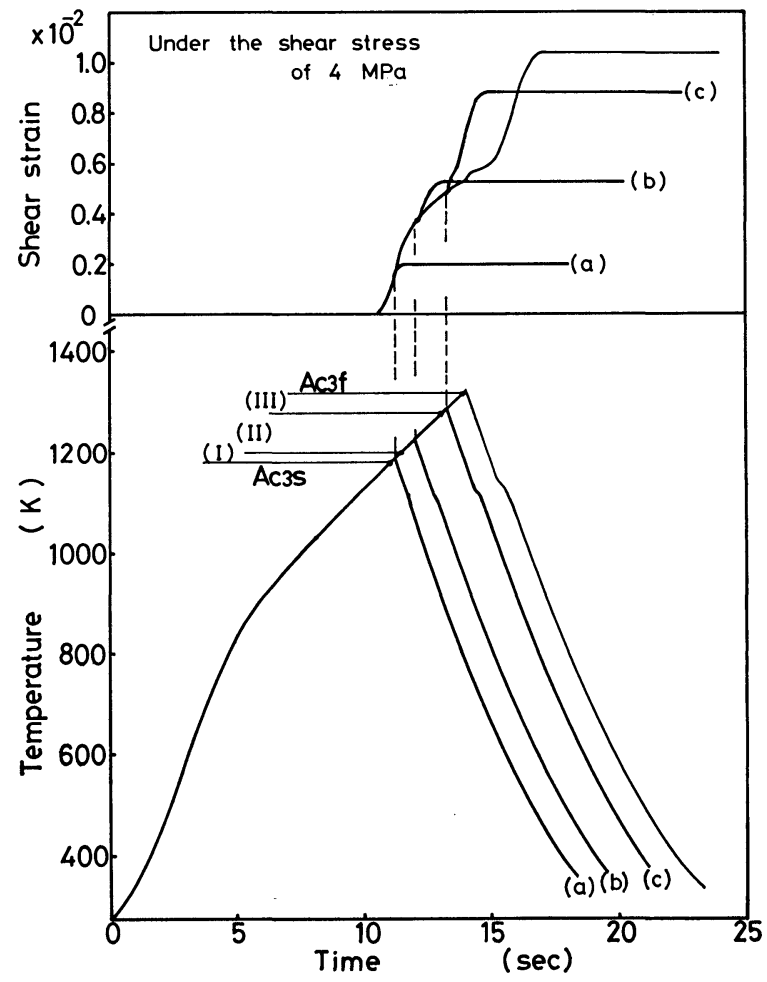

Fig. 12. Rapidly heated to various temperatures $T_{\max }$ and rapidly cooled cycle. Induced strain curves correspond with each temperature cycle. 
12(b)) and then rapidly cooled. The surface morphology, as shown in Fig. $15((a),(b))$, exhibits the trace of partial $\alpha-\gamma-\alpha$ transformation and the front of
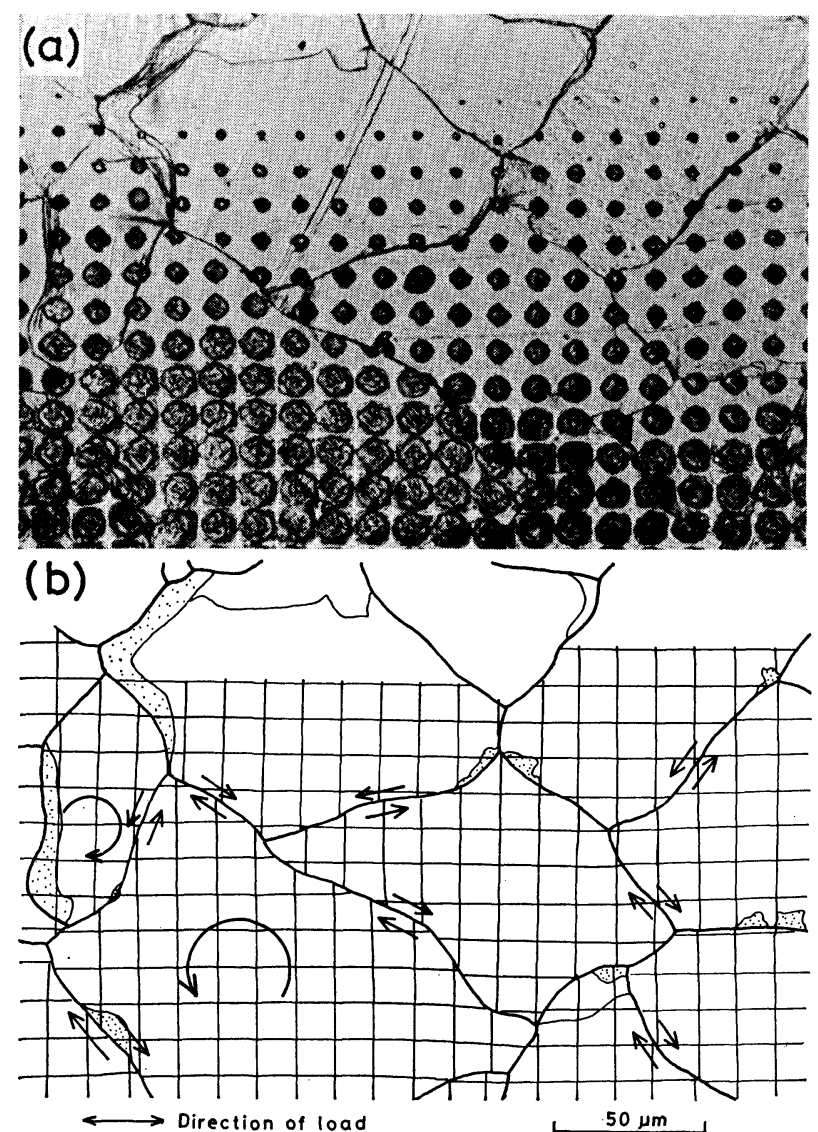

Fig. 13. Microstructure after rapid heating to a temperature just above $A c_{3}$ s and rapid cooling under a tensile stress of $30 \mathrm{MPa}$ in pure iron: grid points are spaced at $12.7 \mu \mathrm{m}$ intervals.
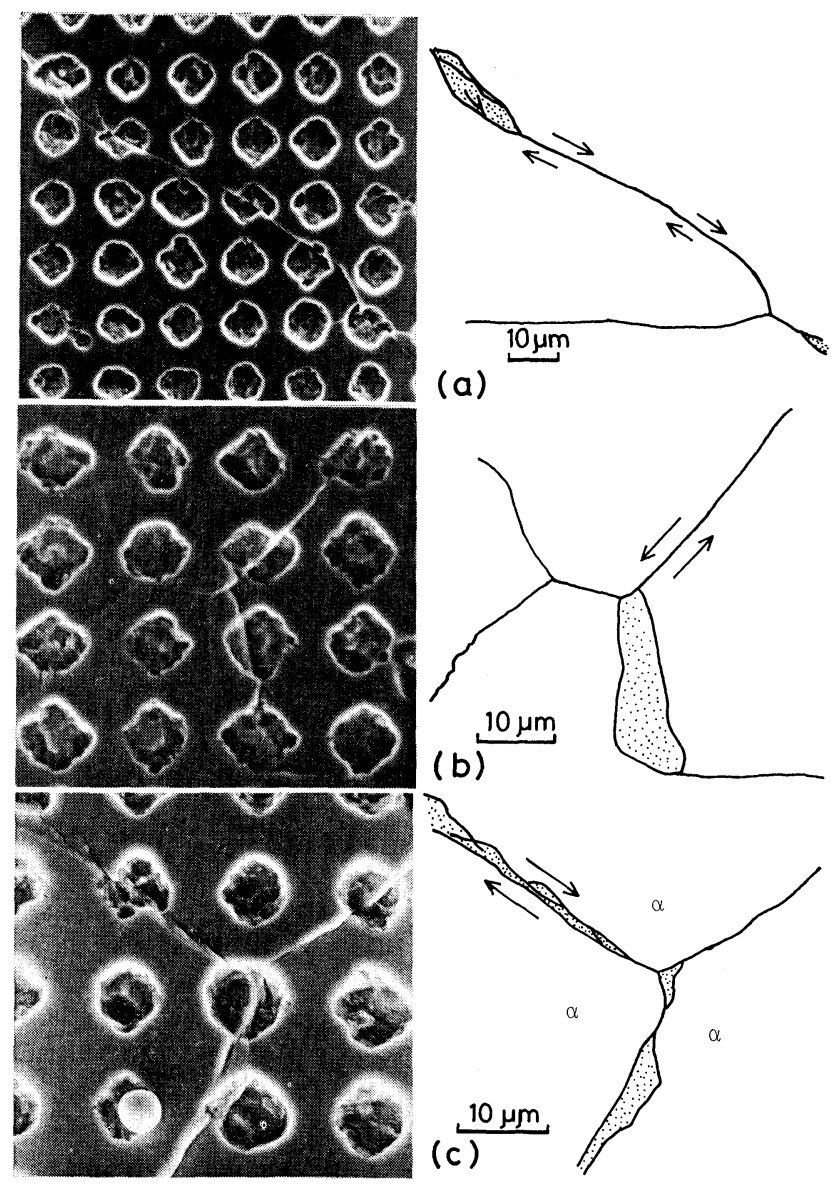

(b)

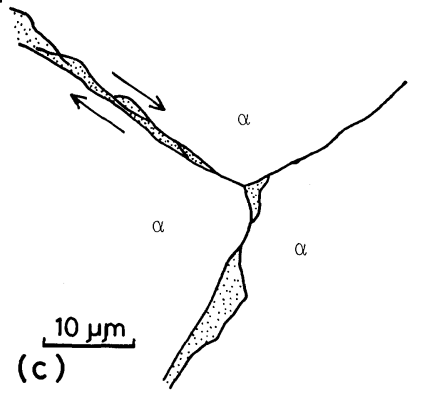

Fig. 14. Microstructure after rapid heating to a temperature just above $A c_{3}$ s and rapid cooling under a tensile stress of $30 \mathrm{MPa}$. Growth of austenite grains and sliding deformation are observed along prior ferritic grain boundaries. (a), (b): (a) Before and (b) after heating to a temperature above $A c_{3} \mathrm{~s}$ under a tensile stress of $20 \mathrm{MPa}$

(d): $\quad \alpha-\gamma-\alpha$ transformed region identified with (a) and (b).

Fig. 15.

Grid analysis of transformation superplastic deformation in pure iron.

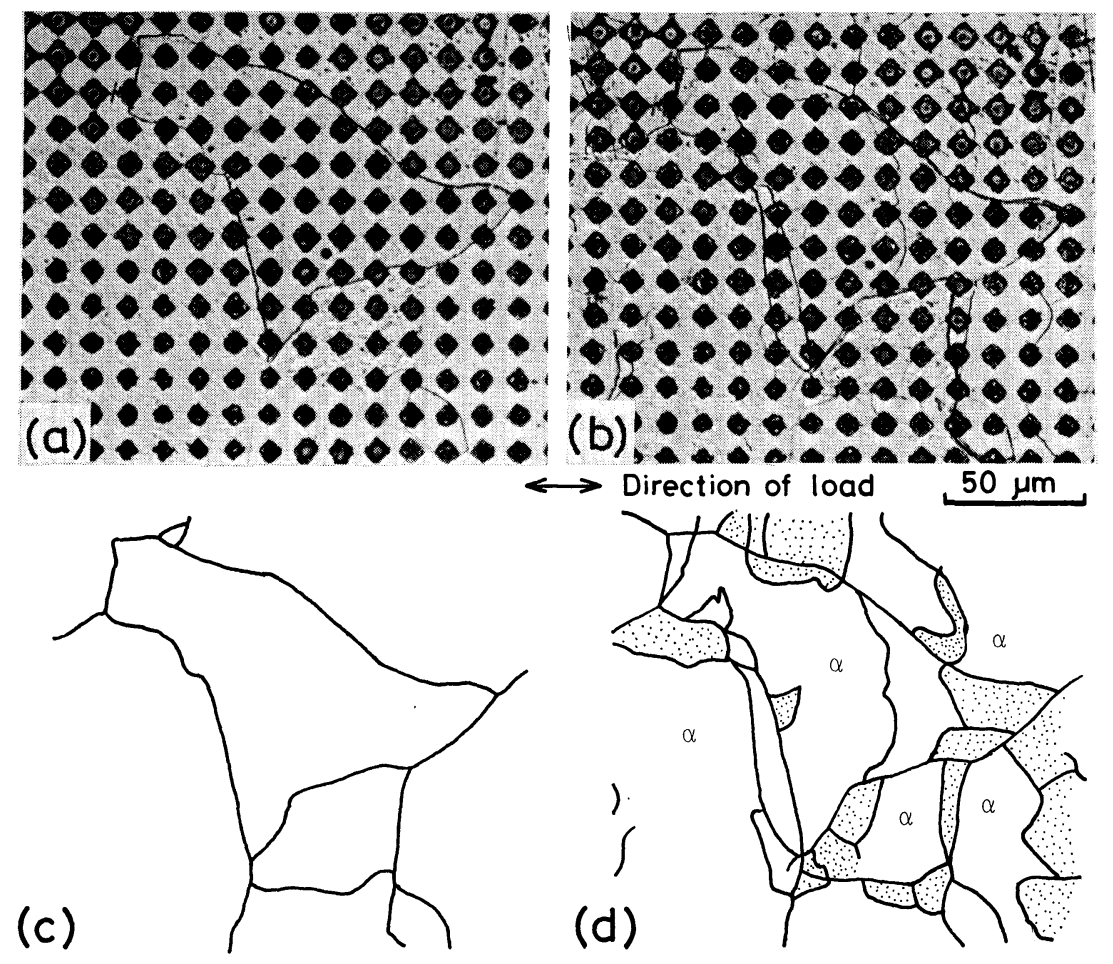




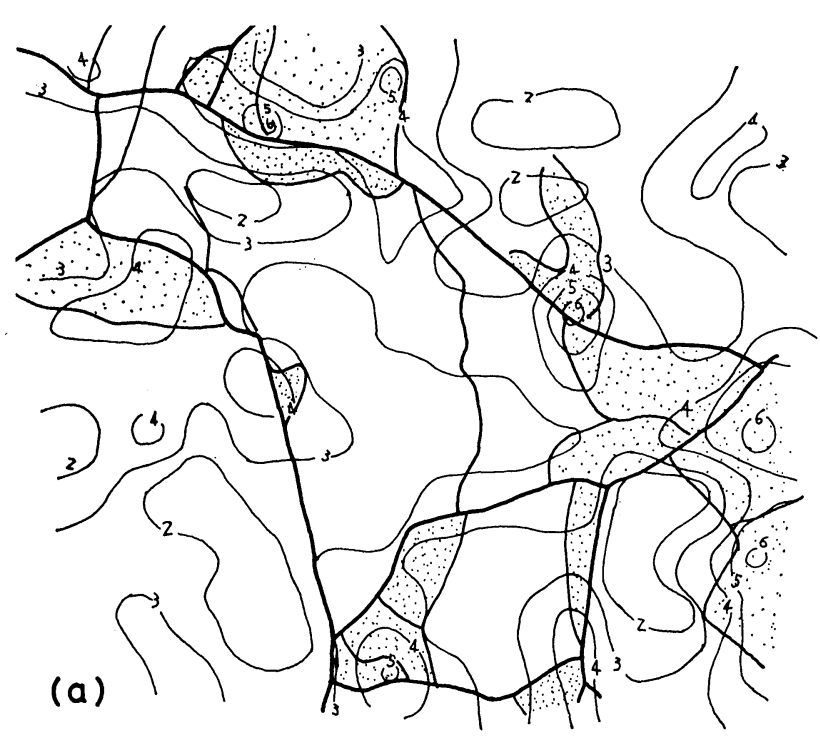

transformation at $T_{\max }$. The grid displacement was analyzed on Fig. 15 and the results are illustrated in Fig. 16.4) Contour lines of an equivalent strain on the basis of Mises criterion are drawn in Fig. 16(a), and the distribution of principal strain vectors $\left(\varepsilon_{1}, \varepsilon_{2}\right)$ is represented in Fig. 16(b). The front of transformed region at $T_{\max }$, obtained from Fig. 15(d), is shown in the same figure.

The dotted area subjected to $\alpha-\gamma-\alpha$ transformation is found to be distributed very similarly to the area where the values of equivalent strain are high. This evidence indicates that the expansion of a strained area is accompanied with the growth of austenite grains in polycrystalline structures due to the progress of $\alpha-\gamma$ transformation.

With reference to the distribution of principal strains as shown by the dotted area in Fig. 16(b), the axis of principal strain $\varepsilon$ is inclined and determined to be about $45^{\circ}$ to the direction of transformation front, as shown in Fig. $17((\mathrm{a}),(\mathrm{b}))$. This suggests that the grain boundary sliding mechanism is dominant on the transformation front during instantaneous phase transformation.

\section{Discussion}

Transformation superplasticity is characterized by the $m$-value of about 1 in the equation $\sigma=k \dot{\varepsilon}^{m}$. When $m$ is 1 , the deformation exhibits a Newtonian viscous flow. In a previous paper ${ }^{3)}$ on the micro-grid analyses of transformation superplasticity, it was found that:

(i) the transformation superplastic deformation was incompressible and homogeneous on the basis of analysis of the trace of grid points during the propagation of deformation, and

(ii) the deformation mode was different from the modes of both plastic deformation at room temperature and creep deformation at a high temperature. Further analyses of macroscopic deformation were made under tensile stresses and torsional stresses. ${ }^{5)}$

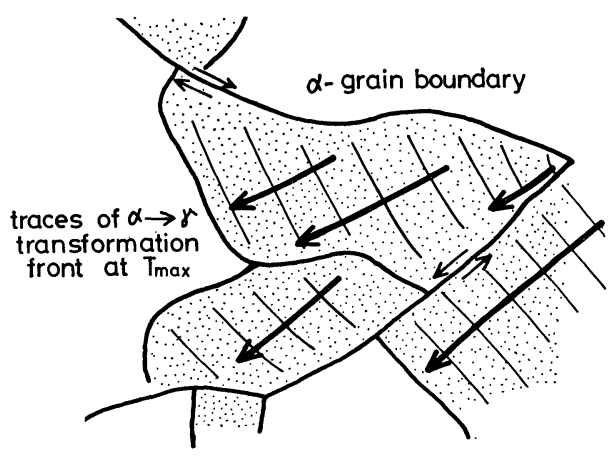

(a) mation. Distribution of (a) equivalent strain $\varepsilon_{\text {eq }}$, (b) principal strain $\varepsilon_{1}$ and $\varepsilon_{2}$.
Fig. 16. Grid analysis of transformation superplastic defor- 
As a result, the three times-law of viscosity, $\lambda=3 \eta(\lambda$ : normal viscosity, $\eta$ : shear viscosity), in other equations, $\bar{\sigma}=3 \tau$ or $\overline{\dot{\varepsilon}}=\dot{\gamma} / 3$ ( $\bar{\sigma}$ : effective stress, $\tau$ : shear stress, $\overline{\dot{\varepsilon}}$ : effective strain rate, $\dot{\gamma}$ : shear strain rate) were found. These findings indicate that transformation superplastic deformation are quite similar in character to viscous flow rather than plastic deformation.

On the other hand, the initial stress is reduced to zero as the transformation proceeds during stress relaxation tests at a constant torsional strain. ${ }^{6)}$ This suggests that liquid-visco-elasticity occurs during the phase transformation, but not firmo-visco-elasticity. The dynamic visco-elastic behavior under transformation superplasticity was confirmed by an experiment of ultrasonic attenuation ${ }^{\text {7) }}$ and the internal friction by means of a torsionally forced vibration method. These facts indicate that a viscous-liquid structure might appear transitorily at an $\alpha / \gamma$ transformation interface during transformation.

In the present study, the movement of an $\alpha / \gamma$ transformation interface and the straining behavior during transformation were investigated in terms of the characteristic deformation in polycrystalline structures. First of all, the transformation process was classified into (I) initial stage, (II) intermediate stage, and (III) final stage, from the dilatometry and superplastic straining behavior.

In the stage (I), austenite grains nucleate at ferrite grain boundaries and triple points. The grains grow along the prior ferrite grain boundaries. In consequence, chains of austenite grains are formed to surround the prior ferrite grains. If the elastic viscousliquid structure appears at the $\alpha / \gamma$ interfaces during transformation, the binding force among ferrite grains might be relaxed, resulting in promotion of slides on the interface along prior ferrite grain boundaries and grain rotations and consequently in progress of superplastic deformation (see Figs. 13 and 14).

In the stage (II), the movement of transformation interface during propagation of austenite grain growth was speculated on the basis of the results in Chap. III. 1 and was represented in Fig. 17(b) along with the distribution of the principal strains. In the figure, the principal strain is found to make $45^{\circ}$ to the tangent at the instantaneous $\alpha / \gamma$ interface. This relationship indicates that the superplastic deformation process might be caused by the slip mechanism acting on the transformation interface. As the transformation proceeds, the transformation interface migrates forward, and while the superplastically strained area might simultaneously expand, resulting in the accumulated strain left in the previously transformed region. These deformation mechanism was confirmed in the previous experiments in which the continuous microscopic observation and strain analyses were carried out on the superplastic deformation during $A c_{3}$ diffusional transformation in a $0.1 \% \mathrm{G}$ steel. ${ }^{8)}$ The superplastic strain is generated on the sweeping interface, but is not generated in the non-transformed ferrite region as shown in Figs. 10 and 15, and in the previously transformed austenite region. ${ }^{8)}$ Hence, these facts suggest that the characteristics of macro- scopic deformation similar to a Newtonian viscous flow will be due to the characteristics of the sweeping interface. On the other hand, an $(\alpha+\gamma)$ duplex in equilibrium during isothermal soaking induced no superplastic strain. ${ }^{2)}$ However, above the critical stress of superplastic deformation of $20 \mathrm{MPa},{ }^{4)}$ a significant strain was found to be induced by slip in a grain as shown in Fig. 10. Consequently, the macroscopic deformation became to be similar to creep deformation and the $m$-value became to be lower than 1 .

As reported in the previous studies ${ }^{3)}$ regarding the stage (III), transformation interfaces were brought in contact on migration. Austenite grain boundary was newly formed, and subsequently to develop a stable grain boundary network after grain boundary migration. At the same time, superplastic deformation is caused by the sliding along the austenite grain boundary.

With regard to the characteristics of deformation in a polycrystalline structures, inhomogeneous deformation was found from the strain analyses, as shown in Figs. 13 and 16. Generally, to continue deformation without any cracks or voids in a polycrystalline structures, the principal mechanisms such as grain boundary sliding or grain rotation should be accompanied with some accomodation mechanisms that can release a local strain concentration and maintain the morphological continuity of aggregates. In ultra-fine grain superplasticity, some accomodation processes were proposed by Ashby and Verral, ${ }^{9)}$ Gifkins ${ }^{10)}$ and other investigators.

In the present study, transformation interfaces exhibited some distinctive behavior. Under a tensile load, austenite grain growth was found to progress anisotropically and the morphology of the interfaces tended to be straight in trace, as shown in Figs. 8 and 10. In Fig. 18(a), an austenite grain grows smoothly in one direction. On the contrary, in Fig. 18(b), the surface relief indicates the successive nucleation of austenite grains during forward sweeping of the interface during $A c_{3}$ transformation. These observations indicate that:

(i) the transformation interface might have a great mobility due to its viscous-liquid structure, and

(ii) in the transformation superplasticity, the transformation interface may play a principal role in the deformation mechanism as well as the accomodation mechanism.

\section{Conclusion}

Microstructural changes during a transformation superplastic deformation process were observed under the specially designed high temperature microscope and strain analyses by a micro grid technique were carried out in pure iron. The results are as follows:

(1) Processes of $A c_{3}$ transformation during heating at a rate under $50 \mathrm{~K} / \mathrm{s}$ include the nucleation of austenite grains at the prior ferrite grain boundaries and triple points, and the subsequent grain growth into the prior ferrite matrix. The growth of austenite grains is not always isotropic under a tensile stress, and an $\alpha / \gamma$ transformation interface tends to be 


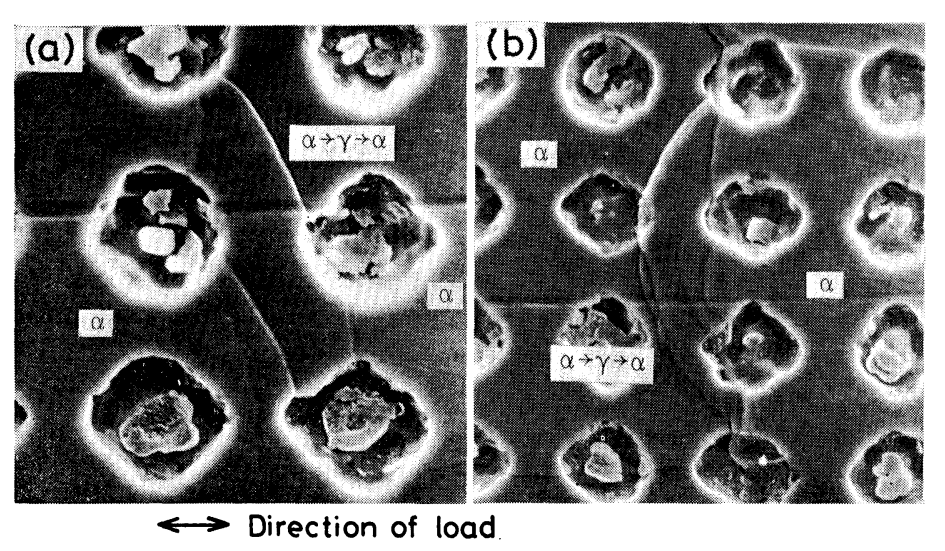

Fig. 18.

Changes of specimen surface relief after heating to a temperature above $A c_{3}$ s point under a tensile stress of $20 \mathrm{MPa}$ in pure iron.

\section{REFERENCES}

straightly aligned.

(2) In the initial stage of transformation, superplastic deformation is induced by the sliding on $\alpha / \gamma$ transformation interface along prior ferrite grain boundaries and the grain rotation, associated with the growth of austenite grains surrounding ferrite grains.

(3) In the intermediate stage of transformation, the sliding deformation is caused on the sweeping transformation interface associated with the progress of austenite grain growth, and the accumulated strain by the sliding is concentrated in the previously transformed regions.

(4) These observations indicate that the sliding mechanism on the migrating transformation interface is a principal mechanism governing the transformation superplasticity.
1) N. Iguchi, Y. Oka and Y. Saotome: J. Jpn. Inst. Met., 38 (1974), 725.

2) N. Iguchi, Y. Oka and Y. Saotome: J. Jpn. Inst. Met., 39 (1975), 357.

3) Y. Saotome and N. Iguchi: Proc. 22nd Jpn. Congr. Mater. Res., 22 (1979), 7.

4) N. Iguchi: J. Mater. Sci. Soc. Jpn., 17 (1980), 103.

5) T. Nishihara, K. Asami and N. Iguchi: J. Jpn. Inst. Met., 41 (1977), 188.

6) T. Nishihara and A. Takase: J. Jpn. Inst. Met., 41 (1977), 751.

7) N. Iguchi, T. Nishihara, H. Takeuchi, E. Nakamura and A. Sakayori: Corrected Abstracts of the 1977 Spring Meeting of Japan Inst. Met., JIM, Sendai, 279.

8) Y. Saotome, K. Kubo and N. Iguchi: Proc. 24th Jpn. Congr. Mater. Res., 24 (1981), 17.

9) M. F. Ashby and R. A. Verral: Phil. Trans. Roy. Soc. (London) A, 288 (1978), 59.

10) R. G. Gifkins: Metall. Trans., 7A (1976), 1225. 